\title{
Inadvertent Insertion of Hemodialysis Catheter into Brachiocephalic Trunk During Cannulation of Internal Jugular Vein: Management Strategies
}

\author{
Chinmaya Nanda ${ }^{1}$ Vinit Garg ${ }^{1}$ Ajmer Singh ${ }^{1}$ Yatin Mehta ${ }^{1}$ \\ ${ }^{1}$ Department of Cardiac Anaesthesia, Institute of Critical Care and \\ Address for correspondence Ajmer Singh, MD, Department of \\ Anesthesiology, Medanta-The Medicity, Sector-38, Gurugram, \\ Haryana, India \\ Cardiac Anaesthesia, Institute of Critical Care and Anesthesiology, \\ Medanta-The Medicity, Sector-38, Gurugram-122001, India \\ (e-mail: ajmersingh@yahoo.com).
}

J Card Crit Care 2021;5:246-248.

\author{
Abstract \\ Keywords \\ - central venous \\ catheter \\ - hemodialysis catheter \\ - inadvertent arterial \\ cannulation
}

Central venous catheter insertion is a commonly performed procedure in the operating room and intensive care unit. It is associated with many complications, some of which may be life-threatening. We report here an accidental insertion of a hemodialysis catheter into the brachiocephalic trunk, which was successfully removed by an endovascular technique.

\section{Introduction}

Insertion of the central venous catheter (CVC) is a commonly performed procedure in the operation theater and intensive care setting. The common sites of insertion of CVC are the internal jugular vein (IJV), subclavian vein, and femoral vein. Traditionally, the insertion of CVC into an IJV is performed by anatomic landmark technique. During access by anatomic landmark method, inadvertent arterial puncture occurs in up to $11 \%$ of patients and misplaced large-bore arterial cannulation occurs in 0.1 to $0.8 \%$ of patients. ${ }^{1,2}$ Real-time ultrasound (US)-guided cannulation is associated with a higher success rate and lesser complications compared with the anatomic landmark method. ${ }^{3}$ Use of US, however, does not eliminate the risk of arterial puncture, and the catheter can still be inadvertently placed into the arterial system. We report here a case of inadvertent insertion of a hemodialysis catheter into the brachiocephalic trunk during cannulation of the right IJV and its subsequent management.

\section{Case Report}

A 62-year-old patient was admitted to the emergency ward with a history of fever, shortness of breath, and altered mentation for 7 days. He was treated as a case of lower respiratory tract infection with respiratory failure. The next day, because of worsening respiratory failure, altered sensorium, and a decrease in arterial oxygen saturation to $85 \%$, the patient was intubated and put on mechanical ventilation. Subsequently, the patient developed hypotension, need for vasopressors, acidosis, oliguria, and hyperkalemia. Nephrology consultation was taken, and they suggested hemodialysis.

After preparation of the neck, using real-time ultrasonographic guidance $(7.5 \mathrm{MHz}$ linear array probe, short-axis view), right IJV was punctured with a $16 \mathrm{G}$ needle. Venous cannulation was confirmed with the aspiration of darkcolored blood in the syringe and nonpulsatile blood flow in the puncture needle. The tip of the guidewire was seen in the IJV in a short-axis view on ultrasound (US). An $11.5 \mathrm{Fr}$ published online January 16, 2022
DOI https://doi.org/ 10.1055/s-0041-1739527. ISSN 2457-0206. (c) 2022. Official Publication of The Simulation Society (TSS), accredited by International Society of Cardiovascular Ultrasound (ISCU). All rights reserved.

This is an open access article published by Thieme under the terms of the Creative Commons Attribution-NonDerivative-NonCommercial-License, permitting copying and reproduction so long as the original work is given appropriate credit. Contents may not be used for commercial purposes, or adapted, remixed, transformed or built upon. (https://creativecommons.org/ licenses/by-nc-nd/4.0/)

Thieme Medical and Scientific Publishers Pvt. Ltd., A-12, 2nd Floor, Sector 2, Noida-201301 UP, India 


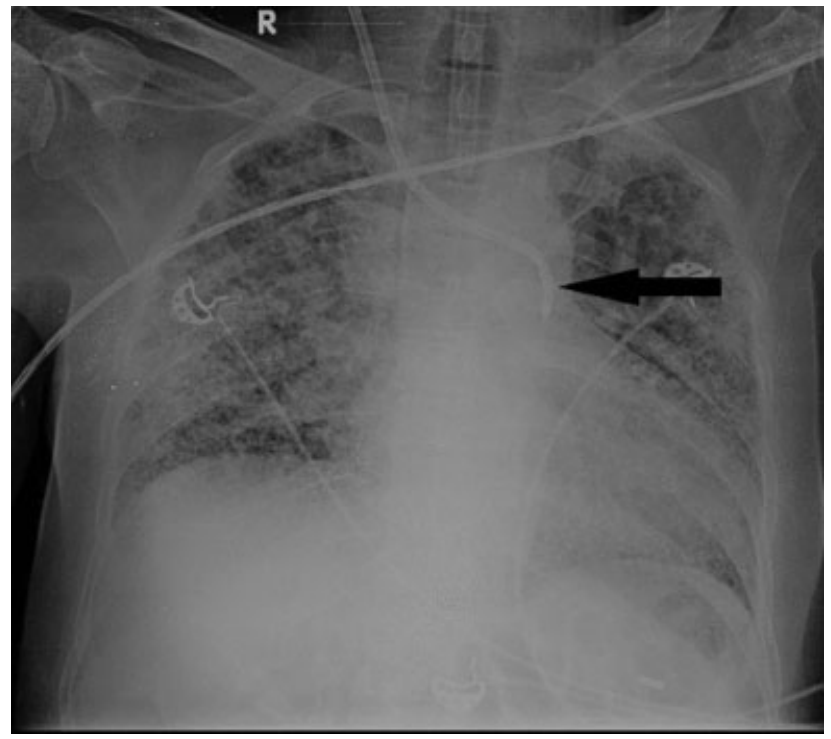

Fig. 1 Chest X-ray showing the hemodialysis catheter lying in the arch of aorta (arrow).

hemodialysis catheter was inserted into IJV by the Seldinger technique. The US was not used in real-time during the insertion of the hemodialysis catheter. Before initiation of hemodialysis, the chest X-ray showed the hemodialysis catheter lying in the arch of the aorta (-Fig. 1). Arterial cannulation was further confirmed by pressure waveform and blood gas analysis. The misplaced catheter was kept in situ, and vascular surgery opinion was taken. Computed tomography angiography (CTA) of head and neck vessels was performed to delineate the course of the catheter. The dialysis catheter was seen coursing from the right side of the neck to the brachiocephalic trunk and the aortic arch ( - Figs. 2A, B). The patient was taken into the hybrid operation theater, and an angiogram was performed through the hemodialysis catheter. The hemodialysis catheter had traversed the posterior wall of the IJV and entered the brachiocephalic trunk. A guidewire was passed through the hemodialysis catheter, followed by removal of the catheter over the guidewire. Two percutaneous closure devices (Perclose ProGlide device, Abbott Vascular, Santa Clara, California, USA) were passed over the wire and deployed to seal the puncture site. Repeat angiogram showed no leak or pseudoaneurysm formation at the vascular device site. Subsequently, the femoral site was chosen for insertion of the hemodialysis catheter, but the patient succumbed 3 days later following a middle cerebral artery infarct.

\section{Discussion}

We assume that ultrasonographic confirmation of the guidewire in the proximal segment of the vein does not always exclude accidental arterial cannulation and, in fact, may impart a false sense of security. Sometimes, the needle can move into the artery during insertion of the guidewire. If an
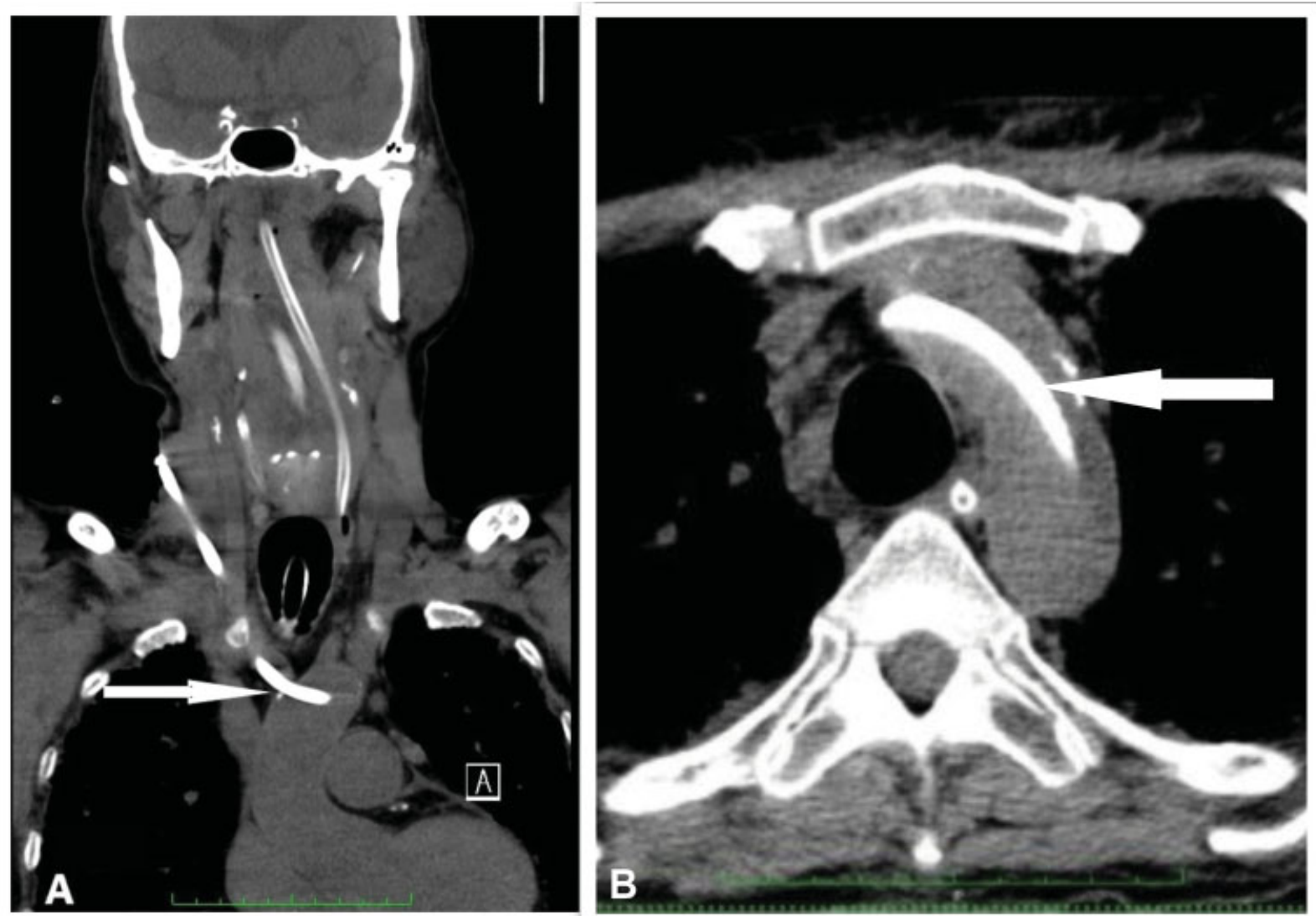

Fig. 2 (A) Computed tomography angiogram (CTA) showing hemodialysis catheter coursing from the right side of the neck into the brachiocephalic trunk and the aortic arch (arrow). (B) Hemodialysis catheter lying in the aorta (arrow). 
US-guided technique is used for CVC insertion, it is essential to reimage the entire vein in both short axis and long axis, and verify that the guidewire lies in the vein before dilatation and railroading of the catheter. ${ }^{4} 2012$ guidelines from the American Society of Echocardiography and the Society of Cardiovascular Anesthesiologists strongly recommend the use of real-time US for CVC placement in the IJV (category A, level-1 evidence) ${ }^{5}$

Unrecognized arterial cannulation with subsequent dilatation and catheter placement can be associated with lifethreatening hemorrhage and neurological complications. There is a potential risk of developing stroke after manual compression following removal of the large-bore catheter. Adverse neurological events most probably result from thrombus formation around the hemodialysis catheter which can migrate later, leading to stroke. Arterial catheter placements have been managed by the following three methods: (i) catheter removal and external compression (pull and pressure method),(ii) surgical exploration and direct arterial repair, and (iii) endovascular intervention. Removal of the arterial catheter and external compression should be performed only if the artery is easily accessible and compressible. The subclavian artery and brachiocephalic trunk, being posterior to the clavicle and sternoclavicular joint, respectively, are inaccessible and incompressible. Compression of the carotid artery carries the risk of cerebral ischemia and dislodgement of emboli. Although open surgical repair of blood vessels supplying cerebral circulation is the standard of care, comorbidities in these patients often make traditional surgical approaches prohibitive. If thoracic surgery has to be performed for other indications, surgery appears to be the best choice to remove the catheter from the brachiocephalic trunk and prevent thromboembolic complications. In the absence of other indications for surgery, an attempt to remove the catheter by endovascular intervention is reasonable, avoiding thoracotomy and possible clavicular or rib resection.

Guilbert et al reported that $47 \%$ of the patients treated by pull and pressure method suffered major complications such as hematoma formation, arteriovenous fistula formation, pseudoaneurysm formation, airway obstruction, and stroke. ${ }^{6}$ They have proposed an algorithm for the management of cervical or thoracic arterial injury with a large-bore catheter. Removal of the large-bore catheter from the brachiocephalic trunk or aorta can lead to perforation of the aorta, massive hemorrhage, cardiac tamponade, and even death. Such patients should be managed by either surgical or endovascular repair. Endovascular methods of arterial injury repair include stent-grafts and collagen-based or suture-based closure devices. Collagen-based devices are designed to occlude an 8 Fr or smaller puncture site. For larger sheaths, suture-based devices such as Perclose device are preferred. The incidences of embolic and thrombotic complications of collagen-based devices range from 0 to $0.5 \%$ and 0 to $1.4 \%$, respectively. ${ }^{7}$ An extensive search of literature regarding the incidence of breakage and embolism of Perclose devices yielded only two results. ${ }^{8,9}$ In both the cases, the embolized device could be retrieved with snare, without any complication. Jahromi et al have reported thromboembolic stroke after percutaneous treatment of inadvertent subclavian artery placement of a CVC. ${ }^{10}$ There have been many reports of the successful use of endovascular closure devices for the management of misplaced arterial catheters during CVC placement. ${ }^{11,12}$ This therapy, however, requires further research and analysis for short- and long-term neurological complications.

This case demonstrates that inadvertent arterial cannulation can occur with CVC placement despite US guidance. Using US to assess anatomy before venous puncture and realtime ultrasonography throughout the entire procedure can reduce such complications. A CTA is suggested to define the extent of arterial injury, especially when the insertion site is not visible. An urgent surgical or endovascular intervention seems to be the safest approach.

Conflict of Interest

None declared.

\section{References}

1 Golden LR. Incidence and management of large-bore introducer sheath puncture of the carotid artery. J Cardiothorac Vasc Anesth 1995;9(04):425-428

2 McGee DC, Gould MK. Preventing complications of central venous catheterization. N Engl J Med 2003;348(12):1123-1133

3 Randolph AG, Cook DJ, Gonzales CA, Pribble CG. Ultrasound guidance for placement of central venous catheters: a metaanalysis of the literature. Crit Care Med 1996;24(12):2053-2058

4 Bowdle A. Vascular complications of central venous catheter placement: evidence-based methods for prevention and treatment. J Cardiothorac Vasc Anesth 2014;28(02):358-368

5 Troianos CA, Hartman GS, Glas KE, et al; Councils on Intraoperative Echocardiography and Vascular Ultrasound of the American Society of Echocardiography Society of Cardiovascular Anesthesiologists. Special articles: guidelines for performing ultrasound guided vascular cannulation: recommendations of the American Society of Echocardiography and the Society Of Cardiovascular Anesthesiologists. Anesth Analg 2012;114(01): 46-72

6 Guilbert MC, Elkouri S, Bracco D, et al. Arterial trauma during central venous catheter insertion: Case series, review and proposed algorithm. J Vasc Surg 2008;48(04):918-925, discussion 925

7 Hoffer EK, Bloch RD. Percutaneous arterial closure devices. J Vasc Interv Radiol 2003;14(07):865-885

8 Kang K, Ferraro R, Petrella R, Kang G. Perclose Closure Device breakage and embolization during deployment followed by retrieval with snare. Indian Heart J 2015;67(Suppl 3):S36-S38

9 Giniyani LL, Rana YP, Hanumanthu BK, Chan D, Kwan TW. Perclose ProGlide embolization as a complication: case report and review of literature. Future Cardiol 2021 (e-pub ahead of print). Doi: 10.2217/fca-2020-0154

10 Jahromi BS, Tummala RP, Levy EI. Inadvertent subclavian artery catheter placement complicated by stroke: endovascular management and review. Catheter Cardiovasc Interv 2009;73(05): 706-711

11 Wallace MJ, Ahrar K. Percutaneous closure of a subclavian artery injury after inadvertent catheterization. J Vasc Interv Radiol 2001; 12(10):1227-1230

12 Ananthakrishnan G, White RD, Bhat R, Chakraverty S. Inadvertent subclavian artery cannulation: endovascular repair using a collagen closure device-report of two cases and review of the literature. Case Rep Vasc Med 2012;2012:150343 